\title{
Hacia una perspectiva humanística del cambio climático global
}

\section{On the way to a humanistic perspective of global climate change}

\author{
Heidy Vega García \\ Universidad Nacional, Costa Rica \\ Heredia, Costa Rica \\ heidy.vega.garcia@una.cr
}

\section{Resumen}

El artículo pretende brindar un panorama general sobre la realidad global y las principales dinámicas relacionadas con el tema del cambio climático, desde un proceso de reflexión humanística, crítica y holística sobre los retos y desafíos actuales que plantea este tema, con el fin de desarrollar una mejor comprensión y un mayor compromiso en la búsqueda de soluciones globales y locales. Además de los aspectos técnicos, la gestión del cambio climático requiere de una perspectiva humanística, capaz de integrar la dimensión ética, social, educativa y cultural, que permita comprender el papel que desempeña el ser humano, su vulnerabilidad, la distribución geográfica del fenómeno, los impactos esperados, los escenarios presentes y futuros (costos y beneficios) y el planteamiento de soluciones posibles, desde las decisiones cotidianas de la ciudadanía, hasta las políticas públicas impulsadas por las instituciones locales, los estados y las organizaciones internacionales. Costa Rica se

ha preocupado y tiene una gran voluntad por mitigar el cambio climático. Sin embargo, el problema es que nuestro país es muy vulnerable a las consecuencias $\mathrm{y}$, en el corto y mediano plazos, se requiere plantear medidas concretas de adaptación que tomen en cuenta sus necesidades reales y que vinculen directamente a la población con participación activa. 
Palabras clave: Humanismo, cambio climático, ambiente, sociedad, planeta.

\begin{abstract}
This article has the intention of providing an overview on the global current status and the main dynamics related to the topic of climate change, from a perspective of a humanistic, critical, and holistic reflection process about the current challenges this subject presents, in order to develop a better understanding and greater commitment in the search for global and local solutions. In addition to the technical aspects, climate change management needs to have a humanistic perspective capable of integrating the ethical, social, educational, and cultural dimensions that will allow the understanding of the role played by human beings, its vulnerability, the geographic distribution of the phenomenon, the expected impacts, the current and future scenarios (costs and benefits) and the proposal of possible solutions, from the every-day decisions of citizens to the public policies promoted by local institutions, states, and international organizations. Costa Rica has been concerned with, and has a strong will in its desire to mitigate climate change. However, the problem is that our country is very vulnerable to the consequences and in the short and medium term it is necessary to propose concrete adaptation actions that take into account its real needs and that directly involve the population in active participation.
\end{abstract}

Keywords: Humanism, climate change, environment, society, planet.

\title{
Introducción
}

Las actuales circunstancias globales nos presentan un panorama alentador, de acuerdo con el concepto tradicional de "progreso económico", considerado como producción, acumulación e intercambio de bienes y servicios. Parece ser que nunca antes en la historia se habían alcanzado etapas tan avanzadas de desarrollo científico-tecnológico, económico y social. Sin embargo, desde el punto de vista planetario, es evidente que todo este "progreso" ha generado muchas externalidades negativas, que se reflejan tanto en el nivel local como en la propia dinámica de la Tierra.

Desde hace más de 40 años, la ecología se ha constituido en una disciplina con características políticas, que se relacionan directamente con otros campos del saber en el tratamiento de los problemas colectivos derivados de la degradación ambiental. Es decir, en la actualidad se concibe la ecología como pensamiento político-social, que defiende los intereses compartidos ligados con la naturaleza. Debido a esto, no es de extrañar que, después de la Cumbre de Río de 1992, el 
tema ambiental haya tomado relevancia en las agendas internacionales y locales, e involucrado a diversos actores, desde organismos internacionales, gobiernos, organizaciones no gubernamentales, instituciones educativas, empresas y ciudadanos comprometidos.

Cada vez se ha venido generando mayor conciencia sobre el peligro que representan la contaminación del aire y de la atmósfera, la deforestación, el calentamiento global y el deterioro de la capa de ozono, fenómenos que se interconectan en el gran peligro de un cambio climático global irreversible, con consecuencias desastrosas y con un marcado tono apocalíptico, que podría significar la desaparición de todo lo vivo en el planeta. Este peligro ha sido comprendido por grupos de científicos, quienes apoyados por gran cantidad de satélites y estaciones meteorológicas registran y advierten sobre las alarmantes fluctuaciones de temperatura, presión y humedad en la atmósfera, dadas en las últimas décadas.

Estos esfuerzos científicos han sido coordinados, durante muchos años, por la Organización Meteorológica Mundial (OMM) y, recientemente, por el Panel Intergubernamental de Expertos sobre el Cambio Climático (IPCC, por sus siglas en inglés). En consecuencia, líderes y personalidades mundiales nos alertan continuamente, desde diferentes medios de comunicación, sobre los peligros que se ciernen sobre la seguridad climática de la entera humanidad, amenaza catalogada incluso como más peligrosa que el terrorismo.

A pesar de la trascendencia del tema, no todos los líderes mundiales han estado ni están de acuerdo con las evidencias científicas que fundamentan la teoría sobre el cambio climático, e incluso muchos científicos se muestran escépticos ante las causas antropogénicas y las consecuencias reales derivadas de las alteraciones de la temperatura del planeta. En este punto, cabe preguntarse ¿por qué a pesar de la trascendencia de este tema, ha sido tan difícil lograr que todos los líderes políticos se comprometan realmente por frenar este problema?

Hay que destacar que en semanas recientes, de acuerdo con Pereda (2015), Barack Obama sorprendió favorablemente a la opinión pública mundial al indicar que "Somos la primera generación que siente las consecuencias del cambio climático y la última que tiene la oportunidad de hacer algo para detenerlo. Este es uno de esos problemas que por su magnitud, si no lo hacemos bien, no podremos reaccionar ni adaptarnos. Cuando hablamos de cambio climático, existe la posibilidad de llegar tarde". 
Ante tal panorama, también hay que tomar en cuenta el llamado del Papa Francisco, en su encíclica ' Laudato Si', en la cual, de acuerdo con Marirrodriga (2015), señala al cambio climático como uno de los principales retos de la humanidad al denunciar "una relación directa entre destrucción del medio ambiente, pobreza y explotación económica" y al advertir que "no sirve luchar contra uno de estos tres factores si no se atacan los otros". Al mismo tiempo, Marirrodriga (2015) menciona que el Pontífice "alza la voz contra la tecnificación obsesiva y un falso humanismo que, en el fondo, relega a la persona en beneficio de la máquina".

Para comprender mejor la amenaza potencial del cambio climático, a continuación se exponen y se analizan algunas consideraciones básicas, necesarias para contrarrestar la inercia y la mala información que circula en algunos medios de comunicación. Ante la necesidad de que la humanidad conozca bien de qué se trata esta amenaza, se requiere que el cambio climático sea analizado desde una perspectiva humanística, capaz de integrar la dimensión ética, social, económica y científica, y que permita comprender el papel que desempeña el ser humano, la distribución geográfica del fenómeno, los impactos esperados, así como los escenarios presentes y futuros.

\section{La atmósfera: capa protectora de nuestra vida}

La comunidad científica internacional señala que la atmósfera es muy importante para el equilibrio de la Tierra, ya que esta recibe, filtra y distribuye toda la energía que proviene del sol (nuestra principal fuente de calor). En la atmósfera suceden procesos fisicoquímicos que hacen posible la vida en el planeta, entre estos se destaca el ciclo hidrológico. Además de estar formada por oxígeno (gas vital para todos los seres vivos), la atmósfera es el espacio de la dinámica de los vientos que dispersan los diversos compuestos gaseosos de una a otra parte de la Tierra y también está formada por la capa de ozono estratosférico $\left(\mathrm{O}_{3}\right)$ que nos protege de los efectos dañinos de los rayos ultravioleta.

Es claro que, en condiciones de equilibrio, la cantidad de calor necesaria para la vida es generada por el efecto invernadero que evita que la Tierra se enfríe. En este proceso, el dióxido de carbono $\left(\mathrm{CO}_{2}\right)$ juega un papel fundamental para retener el calor proveniente del sol; por lo tanto, la temperatura de la superficie terrestre se debe a la interrelación entre el calentamiento y el enfriamiento, es decir, al balance de radiación solar que permanece en la atmósfera después de ingresar y ser reflejada al espacio. Además, la radiación cubre a todo el planeta, pero se extiende de forma diferenciada ya que, por ejemplo, la zona intertropical 
es la que recibe la radiación solar de forma más directa; esta radiación (en forma de calor) luego se distribuye sobre el resto de la superficie terrestre hacia las zonas polares, por la acción de los vientos y de las corrientes oceánicas. A mayor distancia del trópico, las temperaturas disminuyen.

Glynn y Heinke (1999) señalan que la rotación de la Tierra sobre su eje influye en la dinámica de las diversas corrientes de vientos que enfrían y calientan el planeta. La posición geográfica en la superficie terrestre así como los pisos altitudinales determinan que las diversas zonas del planeta reciban cantidades diferentes de humedad. El relieve afecta la distribución de las lluvias. Finalmente, la interacción de las lluvias con la temperatura, la geografía y la altitud de los territorios influye en las características de los diferentes biomas, grupos de ecosistemas que tienen características que los distinguen unos de otros, por ejemplo, desiertos, pastizales y bosques tropicales. Cada bioma tiene clima, suelos, plantas y animales particulares. Estos biomas también son el hábitat de los seres humanos, quienes llevan a cabo sus actividades vitales de acuerdo con los recursos naturales disponibles.

\section{El cambio climático como "tragedia de los terrenos comunales"}

Cada vez son más las investigaciones que atribuyen al ser humano la responsabilidad de alterar el frágil equilibrio planetario, ya que la atmósfera es contaminada por el exceso de partículas generadas por las actividades humanas (principalmente de las industrias y del transporte), al producir grandes emisiones de gases de efecto invernadero, principalmente, dióxido de carbono $\left(\mathrm{CO}_{2}\right)$, a partir de la quema de combustibles fósiles (petróleo, carbón y gas natural). También se habla del impacto en el efecto invernadero que produce el gas metano, como consecuencia de la ganadería y de actividades agrícolas, como los cultivos de arroz.

Giebler (2009) menciona que los expertos de la NASA nos alertan de que si se sobrepasa la cifra de $440 \mathrm{ppm}$ (partes por millón) de $\mathrm{CO}_{2}$, se corre el riesgo de que este aumento "artificial" de $\mathrm{CO}_{2}$ en la atmósfera intensifique el efecto invernadero "natural" y se cree el así llamado calentamiento global (aumento de la temperatura global que el IPCC estima en cuatro grados centígrados, aunque hay estimaciones diferentes en muchos documentos).

Independientemente de la cantidad exacta de grados en que podría aumentar la temperatura, se estima que este calentamiento anormal amenaza el clima mundial, 
pues, ante un posible derretimiento de las zonas polares y glaciales, el aumento del nivel de los mares sería inminente y pondría en riesgo la seguridad de las zonas costeras e incluso la desaparición de muchas de estas.

Las mediciones de la cantidad de $\mathrm{CO}_{2}$ se han venido tomando desde 1950 y lo que muestran es una tendencia creciente, debido al aumento de la población mundial y a su consecuente aporte a la contaminación atmosférica. El periódico español ABC del 27 de mayo del 2014 publicó que las concentraciones de $\mathrm{CO}_{2}$ en la atmósfera superaron el umbral de $400 \mathrm{ppm}$ en todo el hemisferio norte, desde el mes de abril. Ante este dato, la Organización de las Naciones Unidas (ONU) alertan que la concentración media mundial anual de $\mathrm{CO}_{2}$ superará el umbral de las 400 ppm en el 2015 o 2016.

Hurrell (1992) define a la Tierra como un "Ecosistema único, complejo e integrado dentro de las limitaciones de un sistema político (más de 190 Estados soberanos), caracterizado históricamente por el conflicto". Esta definición, extendida hacia la atmósfera, hace que el panorama sea aún más complejo, ya que esta resulta ser un "terreno comunal" imposible de ser delimitado territorialmente, una de las bases de la unidad administrativa definida como "Estado". La atmósfera es única, rodea a todo el planeta por igual, no se puede aislar ni privatizar, todos los que habitamos la Tierra la compartimos.

El movimiento de rotación terrestre y las corrientes de viento transportan fácilmente la contaminación de un lado a otro. Además, el ciclo hidrológico no conoce fronteras, es global. El sol evapora el agua de los océanos y este fenómeno es planetario. Lo que sucede en la atmosfera afectará sin distinción alguna a los países desarrollados y a los países en vías de desarrollo. Nadie se puede escapar (mientras que la migración a otro planeta no sea posible).

\section{¿Es nuestro planeta un interés compartido para toda la humanidad?}

La dicotomía entre la interdependencia ecológica (un solo planeta, una atmósfera compartida e indivisible) y la fragmentación del sistema político internacional (Estados soberanos territorialmente delimitados con intereses individuales) agrava la gobernabilidad del cambio climático, ya que cada Estado busca siempre lograr su interés individual y su bienestar, lo cual genera, directa o indirectamente, perjuicios para los demás. 
Autores como Stiglitz (2006) consideran que la contaminación que producen los países más desarrollados (como Estados Unidos y China) es la principal causa del calentamiento global, pero que las consecuencias más graves las van a sufrir los países más pobres y vulnerables, como las Maldivas y Bangladesh, que se estima podrían desaparecer injustamente ante el aumento del nivel del mar. En muchos escenarios futuros, se habla de que los más afectados serán los que menos contribuyen con el problema.

Por estas razones, en el contexto internacional la gestión del cambio climático debe ser una prioridad, producto de la acción colectiva y comprometida para garantizar que cuando las acciones de un Estado provoquen efectos negativos para otro, se pueda aplicar algún mecanismo de mitigación que disminuya la afectación, por el bien de todos. A pesar de que parece ser lógico que el bien común sea necesario, en el ámbito internacional existen varios factores que inciden en la falta de acuerdos concretos, por ejemplo: la debilidad o ausencia de instituciones capaces de lograr una verdadera y sólida cooperación internacional, la falta de confianza, la búsqueda de intereses a corto plazo, el cálculo de pérdidas y ganancias, la gran heterogeneidad cultural, política y económica del sistema internacional, la diferenciación entre deberes y derechos compartidos versus independencia y autonomía estatal, la desigualdad de poder y riqueza entre países industrializados y países en vías de desarrollo, contextos y problemáticas diferenciadas, por citar algunos.

Las diferencias de percepción sobre el tema tienen que ver también con el aspecto económico. Se menciona que los países en vías de desarrollo (que aún no logran cumplir la equidad intrageneracional) tampoco pueden pensar en cumplir la equidad intergeneracional, ya que la población que no tiene para comer en el presente difícilmente se preocupe de las necesidades de las generaciones futuras.

Estos países se enfocan en solventar sus problemas de deforestación, desertificación, contaminación y pobreza, y su prioridad es la de controlar el crecimiento poblacional. Por otro lado, se establece que los países desarrollados sí podrían enfocarse en la equidad intergeneracional ya que, una vez satisfechas las necesidades del presente, se podrían preocupar de las necesidades de las generaciones futuras; sin embargo, tampoco todos lo están haciendo. Los principales problemas que deben enfrentar son los desechos tóxicos, la degradación ambiental, la lluvia ácida y su prioridad es la de controlar el consumismo.

Otra de las interrogantes es que la percepción de las limitantes para lograr un consenso y una acción real de mitigación del cambio climático en el ámbito 
internacional es relativa a cada realidad. No se puede pretender comparar un escenario latinoamericano con el de Europa y el de Estados Unidos o con otro con más problemas como el de África. Debido a las diferentes percepciones, es que las soluciones no pueden ser impuestas; es decir, los países "desarrollados" no pueden pretender culpar a los países "subdesarrollados" por sus problemas o imponerles las medidas que se consideran necesarias para solucionar las situaciones negativas, ni viceversa, porque en el escenario ambiental internacional la característica principal es de interdependencia compleja, en la cual el todo es producto de la interacción entre las partes.

En este aspecto, existe otra paradoja: si no hay una culpabilidad que pueda ser probada, entonces no habrá culpables y los delitos se seguirán cometiendo en perjuicio de la totalidad. Estos y otros temas se convierten en parte de las agendas políticas de discusión de los principales foros mundiales; no obstante, la situación del ambiente sigue empeorando y, ante la falta de consensos y compromisos asumidos de forma responsable por parte de los mayores contaminantes y ante la ausencia de una verdadera institucionalidad penal internacional que vele por los problemas ambientales, se trata el tema del ambiente más eficazmente en los ámbitos nacionales, regionales y locales de cada país, pese a ser un tema global.

Desde el establecimiento de la Convención Marco de Cambio Climático en 1992, la Organización de las Naciones Unidas (ONU) regula las negociaciones y los acuerdos internacionales sobre este tema. Aunque en la actualidad existen más de 190 Estados miembros, aún muchos otros Estados no han sido reconocidos por esta organización, además de territorios dependientes y áreas de soberanía especial. Por lo tanto, se puede afirmar que el régimen internacional para la gestión del cambio climático posee serias limitaciones de aplicación, ya que no es totalmente planetario.

Además, los compromisos y las acciones concretas para contrarrestar los efectos del cambio climático se encuentran en instrumentos internacionales, sujetos a la firma y ratificación por parte de las autoridades nacionales, por lo que depende de los intereses políticos "de turno" el interés o no por cumplir los compromisos asumidos por las administraciones y autoridades anteriores. Esto resulta evidente en el fracaso de la implementación real del Protocolo de Kioto.

Otro argumento por considerar es que el Derecho Internacional no tiene poder coercitivo. Por ejemplo, la ONU no puede obligar a un país como Estados Unidos a ratificar el Protocolo de Kioto, porque uno de sus principios básicos es el respeto por la autodeterminación de los pueblos. Cada Estado posee su propia 
forma de gobierno, sus políticas nacionales, estrategias, agendas locales y, en la mayoría de los casos, las acciones concretas están sujetas a la voluntad política de los gobiernos en ejercicio.

Finalmente, la ONU, los Estados reconocidos, los instrumentos firmados y ratificados, las administraciones y políticas gubernamentales y las agendas de acción están sujetos al paso del tiempo, por lo que las condiciones dinámicas y cambiantes del entorno pueden hacer que en el transcurso de los años sean obsoletas y no respondan a las necesidades y exigencias reales. En este sentido, el Protocolo de Kioto, establecido en 1997, entró en vigencia en el 2005 y su vencimiento en el 2012 dejó la sensación de que prácticamente no se logró ninguna de las metas propuestas. En 1997 no se contempló el caso de la industrialización de la República Popular China, ni de la India, ni de Brasil, ni de las emisiones de aviones y barcos fuera de las fronteras nacionales.

Otra de las interrogantes sobre la continuación del Protocolo de Kioto es si la siguiente fase debe operar igualmente o con más rigor, ya que todos los países están muy alejados de su nivel de emisiones en 1990, lo que no es realista pensar que acepten reducir sus índices a niveles inferiores. Este tema se espera que sea discutido por la comunidad internacional que planea la renovación y actualización de las metas del Protocolo de Kioto, durante la celebración de la COP 21 de París (en diciembre del 2015). Además, otros sectores manifiestan la necesidad de iniciar un proceso totalmente nuevo, olvidando a Kioto y dejando atrás un proceso internacional que ha tomado 18 años para desarrollarse, para iniciar uno nuevo a partir de cero, lo que incluso podría generar controversias al seno de la ONU y obstaculizar aún más la posibilidad de consenso y compromiso. Mientras la comunidad internacional no adopte las medidas pertinentes para mitigar el cambio climático, el mundo se expone a diversas consecuencias negativas, que pueden resultar inevitables y catastróficas en los próximos años.

\section{Los desafíos humanísticos y éticos frente al cambio climático global}

Desde una perspectiva humanística, la comprensión del cambio climático global debe partir de algunas premisas éticas y morales, necesarias para el bien de la 
humanidad, pero que no han sido aún implementadas en la práctica. Entre estos imperativos, se destacan:

- Exaltar la concepción del ser humano como parte del ecosistema (y por lo tanto, respetuoso de los procesos naturales), sobre la concepción del ser humano, con capacidad de modificar su entorno para la satisfacción de sus necesidades biológicas, socioeconómicas, culturales e individuales. La comunidad de la vida del planeta debe estar por encima de los intereses egoístas de los individuos.

- Anteponer los aspectos ambientales a los humanos: como ya se explicó. Nuestro planeta es un ecosistema global, único e integrado, que debe prevalecer sobre los diferentes sistemas económicos, políticos y culturales fragmentados, fraccionados y separados por fronteras imaginarias en las que los territorios aplican diversas legislaciones y diversos esquemas de poder. Vivimos en un planeta cuya principal característica es la interdependencia ecológica, un mundo complejo y dinámico, donde la naturaleza no conoce límites. El todo está relacionado con cada una de las partes que lo constituyen y, de la misma forma, cada parte puede ser comprendida a través de su relación con el todo. Es una realidad en la que los procesos ambientales siempre tendrán un carácter global, una cadena de causas y consecuencias infinitas, en estrecha relación con los asuntos humanos, que tienen un carácter secundario.

- Consolidar el movimiento ambientalista para poder mitigar las consecuencias del deterioro ambiental en una sociedad con crecimiento poblacional exponencial, con crecientes patrones de consumismo y uso de los recursos naturales. El ambientalismo debe influir positivamente en la población y lograr que los ciudadanos sean "ecociudadanos" con conciencia y compromiso planetario.

- Aplicar de forma real el principio de precaución y de prevención antes que el de restauración: la precaución exige tomar medidas que reduzcan la posibilidad de sufrir un daño ambiental grave, a pesar de que se ignore la probabilidad precisa de que este ocurra, mientras que el principio de prevención obliga a tomar medidas, dado que se conoce el daño ambiental que puede producirse. El cambio climático es impredecible, pero sus consecuencias podrían estimarse. Ante la incertidumbre, es mejor tener precaución y, ante lo concreto, es necesario prevenir. La restauración o reparación debe verse como la última solución, la cual consiste en "reparar" los daños una vez que estos se producen, pero no es totalmente probable que 
la restauración pueda devolver las condiciones originales del ecosistema antes de su deterioro. Por lo tanto, es mejor la precaución y la prevención, antes que la restauración.

- Responsabilizar a los que causan el cambio climático y ayudar a los que lo sufren: la visión sobre este tema difiere sustancialmente entre quienes lo provocan y quienes, sin tener ninguna responsabilidad, sufrirán las consecuencias más negativas, las cuales pondrán en peligro su propia existencia. También incluye el tema de los que están en capacidad de adaptarse y de los que no. Este fenómeno afecta a grupos humanos diferenciados, caracterizados algunos por fragilidad y marginación. Los que lo causan deben colaborar brindando ayudas a la mitigación y adaptación de los más vulnerables. La solidaridad debe ser un imperativo para la supervivencia de todos.

- Regresar a formas de vida más naturales, que reduzcan previamente los impactos humanos en el ecosistema, pero sin descartar la posibilidad de que la tecnología pueda aportar soluciones novedosas al cambio climático.

- Realizar una medición real de la huella ecológica personal y colectiva. En este argumento surge la preocupación de cómo medirlas y, si es posible reducirlas, cuáles son las formas adecuadas para lograrlo, al considerar que todas las sociedades difieren en sistemas económicos, políticos, culturales, jurídicos, entre otros. Este aspecto debe ser más efectivo para establecer una medición estandarizada, para promover la equidad intra e intergeneracional y contribuir a despejar las grandes dudas, especialmente al establecer prioridades para la satisfacción de las necesidades humanas. Es necesario medir, cuantificar, y establecer parámetros claros y justos sobre los "sacrificios" que deberá realizar la generación actual para heredar el patrimonio planetario a las generaciones futuras. La generación presente es la única responsable, quienes aún no nacen no tienen la culpa.

- Resolver el problema del cambio climático debe ser una cuestión de compromiso y de voluntad ética y no un asunto que requiere de mecanismos coercitivos que aseguren su cumplimiento. Las medidas de mitigación del cambio climático deben ser vistas como un asunto urgente, en el que las soluciones planteadas obedezcan al imperativo moral de salvar a la humanidad y no sean consideradas como castigos o sanciones.

- Evitar que deriven nuevas formas de "corrupción ambiental", ya que algunos han visto este tema como una posibilidad para implementar nuevos negocios, por ejemplo, los mercados de emisiones de dióxido de carbono 
en los que predomina la idea de que "el que contamina, paga". Esta última mentalidad está generando que se considere la venta de certificados de emisión de $\mathrm{CO}_{2}$ en el "mercado negro", como una nueva actividad "ilícita" especulativa que desvirtúa el carácter de urgencia y pone en peligro el tema ético del sacrificio y la solidaridad, ya que con estos certificados los países que contaminan pueden pagar para seguir contaminando.

- Pensar positivamente: es necesario creer que aún estamos a tiempo para actuar, que la humanidad va a reaccionar y detener el deterioro, al asumir responsablemente su compromiso con el planeta y con las futuras generaciones. Los optimistas deben superar a los pesimistas que opinan que ya estamos en un punto de no retorno y que ya no es posible hacer nada, que lo único que se puede hacer es adaptarse a las consecuencias.

- Participar socialmente y liderar como parte de la responsabilidad humana global: para que la acción de todos sea importante en la gestión del cambio climático, los compromisos y sacrificios deben empezar desde las decisiones cotidianas que toma cada persona, hasta las políticas de mitigación y adaptación de las instituciones locales, de los estados y de las grandes organizaciones internacionales y no al contrario. Este ha sido el punto más débil hasta el momento. Parece que el tema se "diluye" en el ámbito internacional debido a todos los elementos explicados. Los planes de gestión deben involucrar a cada ciudadano del planeta con aspectos concretos, que tomen en cuenta sus necesidades y sus posibilidades reales de actuación. El ciudadano promedio desconoce todas las dimensiones de este tema; por lo tanto, debe ser educado, sensibilizado y preparado para actuar. En este aspecto, también es importante el compromiso que asuman las grandes empresas automovilísticas y las industrias más contaminantes.

\section{Situación de Costa Rica ante el cambio climático: la visión de cuatro expertos}

Como parte de las actividades de celebración de la Semana de Humanidades 2015, del Centro de Estudios Generales de la Universidad Nacional, el pasado 19 de agosto del 2015 llevó a cabo el Encuentro "Costa Rica ante el cambio climático: escenarios, desafíos y estrategias para la acción”, con el fin de brindar un enfoque integrador en el análisis del cambio climático global y sus efectos para Costa Rica y compartir resultados de diagnósticos multidisciplinarios y propuestas de acciones tendientes a la minimización de problemáticas locales, relacionadas con el cambio climático global (Vega y Cavallini, 2015). 
Al inicio del evento, se proyectó un vídeo del programa "7 Días", de Teletica Canal 7 de Costa Rica (2014) sobre algunos escenarios y desafíos específicos de los efectos que el cambio climático produce a nuestro país. Posteriormente, se expusieron cuatro conferencias a cargo de expertos del Ministerio de Relaciones Exteriores de la República de Costa Rica, del Programa del Estado de la Nación, de la Universidad Nacional y de la Comisión Nacional de Emergencias (CNE).

En primer lugar, la M. Sc. Giovanna Valverde Stark, Jefa negociadora Convención Marco de Cambio Climático de las Naciones Unidas, de la Dirección General de Política Exterior (Ministerio de Relaciones Exteriores de Costa Rica), compartió sobre aspectos de las negociaciones internacionales, como: la Convención Marco de Naciones Unidas sobre Cambio Climático, el Protocolo de Kioto y las perspectivas hacia un Acuerdo de París de carácter vinculante (diciembre, 2015). También se refirió al compromiso de Costa Rica con la C-Neutralidad para el 2021 y las consultas sectoriales, las estrategias nacionales en mitigación (NAMAS) en café, ganadería y vivienda y el Plan Nacional Energético 20142020 y las estrategias nacionales de adaptación (NAPS) que todos los países de ALC están elaborando.

En segundo lugar, el experto Lenín Corrales Chaves, investigador del Programa del Estado de la Nación, desarrolló la conferencia "Desafíos para enfrentar el cambio climático: una mirada desde el estado ambiental del país" e hizo énfasis en la situación actual del mundo y del país en cuanto al aumento del $\mathrm{CO}_{2}$ atmosférico, de las temperaturas y del nivel del mar. Ante dicho panorama, el investigador explicó los siguientes diez desafíos:

1. El país requiere gestionar la información de manera robusta y adecuada sobre el estado y uso del recurso hídrico.

2. Para mantener o mejorar la resiliencia de los ríos, los embalses, las bahías, los golfos y mares, se requiere mejorar el tratamiento de las aguas servidas.

3. Para mantener o mejorar la resiliencia de las comunidades que viven de la pesca, se requiere una gestión sostenible de los recursos, así como considerar los escenarios de cambio climático.

4. Para lograr una adaptación a nivel territorial, se requiere prestar atención a la cobertura del suelo remanente.

5. La consolidación de las áreas silvestres protegidas es una condición ineludible para la adaptación, basada en ecosistemas. 
6. Las zonas urbanas deben ser incorporadas con prioridad a la adaptación y mitigación al cambio climático.

7. Las zonas costeras requieren con urgencia acciones de ordenamiento territorial

8. La adaptación y mitigación al cambio climático requieren aumentar el desarrollo de la innovación y la inversión en investigación y transferencia de tecnología.

9. El fortalecimiento institucional es una condición básica para la adaptación, basada en ecosistemas.

10. La concientización de los consumidores acerca del cambio climático deberá considerarse como un tema central en los próximos años.

En conclusión, este experto menciona que el país tiene acumulado un enorme camino, en materia de conservación, para implementar la adaptación al cambio climático basada en ecosistemas. Sin embargo, si no se cambia el rumbo en materia de inversión en protección y conservación ambiental, estaremos frente a una enorme incertidumbre de si vamos a lograr ser resilientes a los efectos del cambio climático. El país requiere desarrollar un Plan Nacional de Adaptación al Cambio Climático.

En tercer lugar, el Dr. Jorge Herrera Murillo, Vicedecano de la Facultad de Ciencias de la Tierra y el Mar y Coordinador de Laboratorio de Análisis Ambiental (FCTMEDECA) de la Universidad Nacional, desarrolló la conferencia "Desafíos de la adaptación al cambio climático en el Recurso Hídrico en entes operadores del país". Este experto explicó conceptos básicos sobre el clima, el cambio climático antropogénico y enfatizó algunas evidencias de los efectos observados en los últimos años acerca del aumento de la temperatura, del contenido de vapor de agua en la atmósfera y de la elevación del nivel medio del mar en el mundo.

Posteriormente, se refirió a las tendencias de precipitación y temperatura (estimadas hacia el 2100) en escenarios específicos de Costa Rica, como la Región Central, el Pacífico Norte, el Pacífico Central y la Zona Norte. En dichos escenarios, el aumento de la temperatura es una tendencia general, así como la disminución de las precipitaciones (a excepción del Pacífico Central, que muestra la tendencia hacia el aumento, hasta en un $14 \%$, en la última década del siglo XXI). Ante tal panorama, el experto enfatizó el concepto de vulnerabilidad del recurso hídrico, los efectos del cambio climático en los ecosistemas, en la calidad del agua, en las inundaciones y sequías y la importancia de los entes operadores de sistemas de abastecimiento de agua frente a estos desafíos. También se refirió 
a los costos y a las medidas de adaptación y a los componentes de un Plan de Adaptación para un ente operador.

Y, en cuarto lugar, el M. Sc. Carlos Picado Rojas, Jefe de la Unidad Estratégica, Comisión Nacional de Emergencias, presentó la conferencia "Riesgo a desastres y adaptación al cambio climático", en la cual explicó el concepto de adaptación desde la política nacional de gestión del riesgo al 2030, al indicar que Costa Rica presenta amenazas potenciales, como: inundaciones, inestabilidad de laderas, fallas geológicas, áreas generadoras de flujo de lodo o avalanchas y áreas bajo influencia volcánica, que la hacen muy vulnerable.

Los eventos hidrometeorológicos y geotectónicos significan pérdidas constantes de millones de dólares, mayormente en los sectores de infraestructura vial, sistema eléctrico, agropecuario, vivienda, ríos y quebradas, ambiente, entre otros. de acuerdo con el índice de déficit por desastre (IDD), el país no tendría recursos propios suficientes, o por transferencia y financiación factible, para afrontar las pérdidas y realizar la reposición del "stock" de capital afectado. Los desastres implican una obligación o pasivo contingente no explícito, que puede significar un impacto a la sostenibilidad fiscal, dado que la mayoría de los recursos a los que se podría acceder representan fondos propios y nuevos endeudamientos. El gobierno retiene en gran parte las pérdidas y su financiación representa un alto costo de oportunidad dadas las necesidades de inversión y las restricciones presupuestales existentes.

Este experto mencionó también la necesidad de construir una agenda común entre gestión para la reducción del riesgo y adaptación al cambio climático, ya que el eje de adaptación de la Estrategia Nacional de Cambio Climático es el vínculo que une la gestión del riesgo al tema del cambio climático, pues plantea reducir la vulnerabilidad de la población costarricense, las causas de las pérdidas de vidas humanas y las consecuencias sociales, económicas y ambientales, inducidas por las amenazas de origen natural y antrópico, que afecten el territorio nacional de Costa Rica.

Picado también se refirió al concepto de "resiliencia", entendido como capacidad de un sistema, comunidad o sociedad, potencialmente expuesto a amenazas, para adaptarse, resistiendo o cambiando, con el fin de alcanzar o mantener un nivel aceptable en su funcionamiento y estructura. La resiliencia está determinada por el grado en que el sistema social puede organizarse para incrementar su capacidad de aprender de desastres pasados, a fin de protegerse mejor en el futuro y mejorar las medidas de reducción de los riesgos. En este tema es fundamental la inclusión social, la participación y desconcentración, la educación, el desarrollo 
del conocimiento y la innovación, la sostenibilidad financiera e inversión en infraestructura y servicios, la planificación, y los mecanismos e instrumentos normativos para la reducción del riesgo.

\section{Reflexión final}

Parece que en el siglo XXI el ser humano, "dotado de gran racionalidad", no logra cumplir el compromiso ético con su especie ni con su planeta. En este punto se destaca la urgencia de un cambio "civilizatorio" y necesario para que la humanidad, en su conjunto, se replantee su presente y pueda aspirar a una visión de futuro positiva para nuestra Tierra compartida.

Desde la aparición del ser humano en la Tierra, el desarrollo "civilizatorio" ha sido la pauta. La búsqueda del desarrollo como progreso material, la aplicación de la racionalidad, la cientificidad, la jerarquización y especialización del pensamiento han caracterizado el devenir de las comunidades humanas y dejado cada vez más de lado los aspectos biológicos esenciales.

La gestión del cambio climático requiere de una actuación urgente. El ser humano no puede arriesgarse a ver si la teoría se confirma, si se trata de un asunto de supervivencia que no debe ser postergado. La interacción del clima con el suelo, las plantas, los animales y el recurso hídrico del planeta deben mantenerse inalterados para garantizar las condiciones necesarias para la vida, a pesar de la creciente presión poblacional humana, la desaparición de los bosques y la extensión de cada vez más ciudades contaminadas y calientes. Lastimosamente, el calentamiento global no se puede solucionar con un simple aire acondicionado en nuestros hogares, se trata de un asunto muy serio. Pero hasta que no existan una verdadera conciencia y acción, el peligro no va a desaparecer, aunque muchos lo oculten deliberadamente o lo quieran negar, las evidencias cada vez son más frecuentes, tanto en el nivel local como en el regional e internacional.

Nuestro país es un ejemplo de las regiones más vulnerables al cambio climático y los expertos que compartieron su experiencia en el Encuentro del 19 de agosto alertaron sobre esas señales preocupantes, anteriormente nunca vistas. Son los nuevos desafíos que el siglo XXI nos impone y que como país no se pueden ignorar.

Se puede afirmar que en Costa Rica ha habido gran preocupación y voluntad en querer mitigar el cambio climático. Sin embargo, el problema es que nuestro país es muy vulnerable y en el corto y mediano plazos se requiere plantear medidas concretas de adaptación. Los documentos oficiales como la Estrategia Nacional 
de Cambio Climático y las Tres Comunicaciones Nacionales poseen información muy valiosa que debe ser conocida, analizada y actuada por toda la población. Los escenarios proyectados dentro de 100 años deben inspirarnos a tomar las decisiones antes de que sea tarde. En las actuales circunstancias, adaptarnos parece no ser una opción más, sino la única.

\section{Referencias}

ABC, Periódico Electrónico. (2014). Las concentraciones de $\mathrm{CO}_{2}$ superan las 400 ppm en todo el hemisferio norte. Recuperado de http://www.abc.es/naturalcambioclimatico/20140527/abci-hemisferio-norte-201405271048.html

Giebler, A. (2009). Lo bello y el caos. San José: Editorial UCR.

Glynn, H. y Heinke, G. (1999). Ingeniería ambiental. México: Prentice Hall.

Hurrell, A. \& Kingsbury, B. eds. (1992). The international politics of the environment. Great Britain: Oxford Press.

Marirrodriga, J. (2015). 'Laudato si”: Francisco evoca a Francisco de Asís. Diario Digital El País, 17 de junio. Madrid. Recuperado de http://elpais.com/elpais/2015/06/16/ opinion/1434479145 446806.html

Pereda, C. (2015). Obama: "El cambio climático es la mayor amenaza para nuestro futuro". Diario Digital El País, 4 de agosto. Madrid, España. Recuperado de http://internacional. elpais.com/internacional/2015/08/03/actualidad/1438626306 883702.html

Rubio de Urquia, F. (2006). El cambio climático más allá de Kioto: elementos para el debate. Ministerio del Medio Ambiente. Madrid.

Stiglitz, J. (2006). ¿Cómo hacer que funcione la globalización? Madrid ,Taurus.

Teletica Canal 7. (2014). Programa "7 Días" sobre escenarios y desafios del cambio climático para Costa Rica. Transmitido el 13 de octubre, 2014. San José.

Vega, H. y Cavallini, E. (2015). Urge plan de adaptación a cambio climático. Hoy en el Campus. Boletín Digital de la Universidad Nacional. Octubre, 2015. Recuperado de http://www.hoyenelcampus.una.ac.cr//index.php?option=com $\underline{\text { content } \& \text { task }=\text { view } \& \mathrm{id}=1656 \& \text { Itemid }=50}$

Vega, H. y Cavallini, E. (2015). Síntesis del "Encuentro Costa Rica ante el cambio climático: escenarios, desafios y estrategias para la acción”. Comisión Ambiental del Centro de Estudios Generales, Comisión Difusión del Informe Estado de la 
Nación, Red Carta de la Tierra UNA, Programa UNA Campus Sostenible y Programa Interdisciplinario de Investigación y Gestión del Agua (PRIGA). Universidad Nacional. Costa Rica.

Vega, H. (2011). Centroamérica: un territorio vulnerable con sociedades frágiles: Reflexiones en torno al cambio climático y su relación con el desplazamiento humano. Revista Ístmica 114. Revista. Universidad Nacional, Costa Rica, pp. 69-88. 